\title{
BMJ Open Developing implementation strategies for firearm safety promotion in paediatric primary care for suicide prevention in two large US health systems: a study protocol for a mixed- methods implementation study
}

\author{
Courtney Benjamin Wolk, ${ }^{1}$ Shari Jager-Hyman, ${ }^{1}$ Steven C Marcus, ${ }^{2}$ \\ Brian K Ahmedani, ${ }^{3}$ John E Zeber, ${ }^{4}$ Joel A Fein, ${ }^{5,6}$ Gregory K Brown, ${ }^{1}$ \\ Adina Lieberman, ${ }^{1}$ Rinad S Beidas ${ }^{1}$
}

To cite: Wolk CB, JagerHyman S, Marcus SC, et al. Developing implementation strategies for firearm safety promotion in paediatric primary care for suicide prevention in two large US health systems: a study protocol for a mixedmethods implementation study. BMJ Open 2017;7:e014407. doi:10.1136/ bmjopen-2016-014407

- Prepublication history for this paper is available online. To view these files please visit the journal online (http://dx.doi. org/10.1136/bmjopen-2016014407).

Received 22 September 2016 Revised 27 April 2017 Accepted 24 May 2017

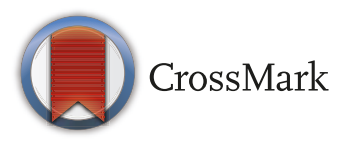

For numbered affiliations see end of article.

Correspondence to

Rinad S Beidas; rbeidas@upenn. edu

\begin{abstract}
Introduction The promotion of safe firearm practices, or firearms means restriction, is a promising but infrequently used suicide prevention strategy in the USA. Safety Check is an evidence-based practice for improving parental firearm safety behaviour in paediatric primary care. However, providers rarely discuss firearm safety during visits, suggesting the need to better understand barriers and facilitators to promoting this approach. This study, Adolescent Suicide Prevention In Routine clinical Encounters, aims to engender a better understanding of how to implement the three firearm components of Safety Check as a suicide prevention strategy in paediatric primary care.
\end{abstract}

Methods and analysis The National Institute of Mental Health-funded Mental Health Research Network (MHRN), a consortium of 13 healthcare systems across the USA, affords a unique opportunity to better understand how to implement a firearm safety intervention in paediatric primary care from a system-level perspective. We will collaboratively develop implementation strategies in partnership with MHRN stakeholders. First, we will survey leadership of 82 primary care practices (ie, practices serving children, adolescents and young adults) within two MHRN systems to understand acceptability and use of the three firearm components of Safety Check (ie, screening, brief counselling around firearm safety and provision of firearm locks). Then, in collaboration with MHRN stakeholders, we will use intervention mapping and the Consolidated Framework for Implementation Research to systematically develop and evaluate a multilevel menu of implementation strategies for promoting firearm safety as a suicide prevention strategy in paediatric primary care.

Ethics and dissemination Study procedures have been approved by the University of Pennsylvania. Henry Ford Health System and Baylor Scott \& White institutional review boards (IRBs) have ceded IRB review to the University of Pennsylvania IRB. Results will be submitted for publication in peer-reviewed journals.
Strengths and limitations of this study

- This study aims to engender a better understanding of how to implement the three firearm components of a universal evidence-based safety intervention (ie, Safety Check) as a suicide prevention strategy in paediatric primary care.

- This study will advance the implementation science literature by using intervention mapping to systematically develop multilevel implementation strategies in partnership with stakeholders.

- The study will be conducted in two large, diverse, and geographically distinct health systems in the USA, and the extent to which results will generalise to other health systems is unknown.

- Both participating health systems are a part of the Mental Health Research Network (MHRN); health systems participating in the MHRN may have unique characteristics that may limit generalisability.

- The firearm components of Safety Check have previously been bundled with other injury prevention strategies; thus, the effectiveness of Safety Checkas a stand-alone firearm safety intervention specifically for suicide prevention in paediatric primary care is unknown.

\section{INTRODUCTION}

The rate of youth suicide has increased steadily over the past 15 years. ${ }^{1}$ The most recent statistics identify suicide as the second leading cause of death among adolescents in the USA, ${ }^{2}$ making this a critical age group to target for suicide prevention strategies. Firearms are the most common and most lethal method of suicide deaths in the USA; risk of suicide is two to five times greater in homes containing a firearm. ${ }^{3}$ This is of great concern given that one in three US homes contain 
a firearm. ${ }^{45}$ Thus, safe firearm practices at home are of paramount importance in reducing death by suicide among youth.

Lethal means restriction refers to the promotion of practices to make the environment safer by reducing access to potentially lethal methods of suicide. Several population-level natural experiments have supported the efficacy of means restrictions. ${ }^{6}$ For example, in the 1960s, the UK substituted domestic gas with a non-toxic alternative, resulting in a $30 \%$ drop in the national suicide rate. ${ }^{78}$ In the 1990s, the Sri Lankan government placed restrictions on the sale of toxic pesticides, the leading suicide method in the country at the time, and overall suicide rates dropped by 50\%. ${ }^{9}$ A 2006 Israeli policy designed to reduce soldier suicide required soldiers to leave their firearms on base during periods of leave, resulting in a $40 \%$ decrease in soldier suicides. ${ }^{10}$

Reducing access to firearms is one promising, although underutilised, suicide prevention strategy. Firearm means restriction strategies include the promotion of safe firearm storage practices as well as gun control legislation to reduce availability to firearms generally and to children in particular. In the USA, broadly reducing access to firearms has been deemed infeasible. ${ }^{11}$ Child access protection laws are associated with modest effects. ${ }^{12}$ Estimates from case-control and simulation studies suggest that a firearm safety intervention implemented in the USA yielding even modest results (ie, only one-fourth of households restrict access to firearms) could lead to 3600-3900 adult lives saved annually. ${ }^{613}$

Although suicide prevention strategies for youth have traditionally been implemented in behavioural health settings, ${ }^{14}$ only one-third of youth receive mental health treatment in the 12 months preceding a suicide attempt. ${ }^{15-17}$ Yet four out of five youth who die by suicide will have visited primary care in the year prior to their death ${ }^{16}$ and $90 \%$ of youth visit primary care annually, ${ }^{18}$ highlighting the advantages of implementing a universal prevention approach in primary care for firearm safety interventions. One intervention, Safety Check, includes a tripronged approach to firearm safety counselling and resulted in safer firearm storage practices in homes with children aged 2-11 years. ${ }^{19}$ Safety Check in its original form includes screening about access to firearms, brief counselling informed by motivational interviewing informed counselling regarding firearm safety and provision of free firearm locks bundled with other injury prevention strategies (eg, use of time out). ${ }^{19}$ In a large cluster-randomised trial of 137 paediatric primary care practices, parents who received the Safety Check intervention reported they were $21.4 \%$ more likely to engage in safe firearm storage practices compared with parents in the control condition at 6-month follow-up. Despite the substantial evidence base, as well as recommendations from the American Academy of Pediatrics ${ }^{20}$ and the Institute of Medicine, ${ }^{21}$ the three firearm components of Safety Check continue to be underutilised in US paediatric primary care clinics. ${ }^{131522}$
To maximise the likelihood that this promising intervention is translated into practice, it is essential to gain a more nuanced understanding of the implementation context from multiple levels. Importantly, it is necessary to understand barriers and facilitators to implementation that relate to characteristics of providers, organisations and the health systems, as well as the states in which they work. These perspectives have been understudied. In the case of firearms, it is also important to obtain the perspectives of both firearm-owning and non-owning stakeholders when possible. ${ }^{23}$ While information can be gleaned from efforts to implement other safety interventions, such as seatbelts, given that doctor-patient firearm conversations have been the topic of legislation in the USA, firearm safety interventions may involve unique barriers. Therefore this formative work is critical to the successful, large-scale implementation of Safety Check.

The Consolidated Framework for Implementation Research (CFIR) provides a context to guide the project. ${ }^{24}$ The CFIR ${ }^{24}$ is an effort to synthesise the many conceptual frameworks in implementation science. ${ }^{25}$ The five major domains that comprise the $\mathrm{CFIR}^{24}$ are (1) intervention characteristics (ie, the features of an intervention); (2) outer setting (ie, the economic, political and social context within which an organisation exists); (3) inner setting (ie, the organisational setting); (4) characteristics of individuals involved in implementation (eg, providers); and (5) the implementation process (see figure 1). In the case of Safety Check implementation, intervention characteristics such as terminology (eg, means restriction vs firearm safety, endorsement of written materials by firearm organisations) may be important. At the provider level, low self-efficacy or lack of knowledge about best practices for firearm safety promotion and/or suicide prevention may contribute to provider reluctance to engage in discussions with patients about firearms. ${ }^{26-29}$ At the inner setting, barriers and facilitators regarding availability of resources (eg, firearm locks, educational pamphlets focused on safe storage of firearms) and organisational support (eg, buy-in from front-desk staff and management) may be present. Outer setting barriers may include lack of policies to support implementation (eg, absence of guidelines about firearm counselling and associated documentation in the electronic health records) and state legislation. For example, the Florida Privacy of Firearm Owners Act, passed in 2011, stipulated that providers must refrain from enquiring about firearm ownership and documenting firearm-related information in patients' medical record except under specific circumstances, such as when the healthcare practitioner determines that this information is relevant to the patient's safety or the safety of others. Of note, despite the stipulation permitting providers to enquire about and/or document firearms-related information when relevant to matters of safety, many providers mistakenly believed that it was illegal to discuss firearms during healthcare visits under any circumstances, ${ }^{30}$ and thus refrained from doing so. Key provisions of this legislation were overturned in 


\section{Intervention Characteristics (i.e., features of Safety Check)}

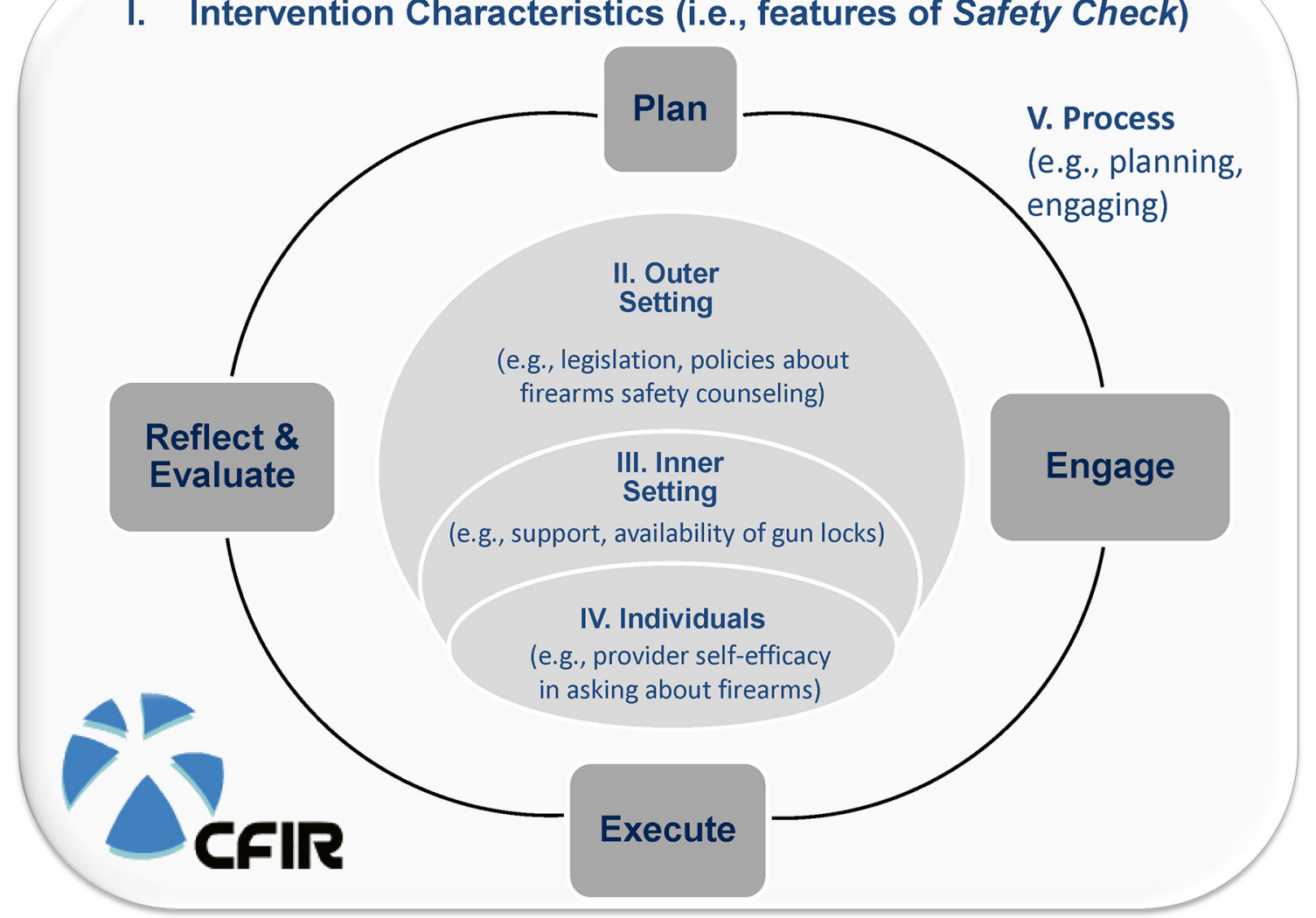

Figure 1 Study guiding framework. Figure adapted from Damschroder. ${ }^{62}$ CFIR, Consolidated Framework for Implementation Research.

$2017^{31}$; however, similar legislation has passed in other states (eg, Montana, Missouri and Minnesota), and more restrictive legislation has been proposed elsewhere (eg, a pending proposal in Ohio prohibits any enquiries related to firearm ownership or possession without exception). ${ }^{30}$ Currently, there are no state or federal laws prohibiting provider questioning or counselling about firearms.

Intervention mapping is a systematic approach to intervention development that has been used to develop a number of effective health-related programmes, including those addressing sex education, ${ }^{32}$ obesity, ${ }^{33}$ cancer prevention, ${ }^{34}$ osteoarthritis and low back pain. ${ }^{35}$ Intervention mapping, used in concert with the CFIR, ${ }^{24}$ can be applied to develop a menu of implementation strategies in a systematic and rigorous way by helping identify who should be involved in adoption, implementation and maintenance of interventions, as well as what specific behaviours they need to engage in. ${ }^{36}$ To date, implementation strategies have not been developed methodically and have lacked rigour in their application. ${ }^{37}$

\section{Objectives and aims}

Our goal in this study (Adolescent Suicide Prevention In Routine clinical Encounters; ASPIRE) is to collaboratively develop implementation strategies in partnership with stakeholders to understand how to most effectively implement a firearm safety intervention in US paediatric primary care (operationally defined as including paediatrics, internal medicine, family medicine and/or adolescent medicine). To date, there has not been a study to systematically examine factors that may impede or facilitate implementation of a firearm safety intervention as a suicide prevention strategy at the provider, organisation and system levels. This formative work is necessary to inform the development of multilevel implementation strategies to reduce death by suicide in youth. The National Institute of Mental Health-funded Mental Health Research Network (MHRN; www.mhresearchnetwork.org), a consortium of 13 healthcare systems across the USA, is an exceptional laboratory with established research infrastructure in which to better understand how to implement a firearm safety intervention in primary care from a system-level perspective.

\section{Aim 1}

We will survey the clinical leadership and providers of 82 primary care practices within two MHRN systems to understand (1) beliefs about the acceptability of screening, brief counselling around firearm safety and provision of firearm locks within paediatric primary care; and (2) use of these practices. Additionally, we will investigate if patient-level, provider-level and system-level characteristics predict acceptability and use of these practices.

\section{Aim 2}

In collaboration with MHRN stakeholders in these two systems, we will use intervention mapping and the $\mathrm{CFIR}^{24}$ 
to systematically develop and evaluate a multilevel menu of implementation strategies for youth firearm safety as a suicide prevention strategy in paediatric primary care.

\section{METHODS AND ANALYSIS}

We began data collection for this project in August 2016. The study is expected to conclude in April 2018.

\section{Setting}

The MHRN is a consortium of 13 public domain research centres based in large not-for-profit healthcare systems covering approximately 13 million lives in 15 US states. The mission of the MHRN is to improve the management of mental health conditions by connecting research, practice and policy. We have recruited two MHRN health systems to participate in project ASPIRE. The Henry Ford Health System (HFHS) has 37 primary care practices and serves 1 million lives annually; $12 \%$ of those individuals are under the age of 18 . Thirty-eight per cent are ethnic minorities, which is important, given evidence that there are widening racial disparities among youth in the USA such that suicide has increased among black children while decreasing among white children. ${ }^{38}$ Baylor Scott \& White (BSW) has 45 primary care practices and serves 630000 lives annually; nearly $20 \%$ of those individuals are under the age of 18 . This system is located in Texas, where proposed legislation prohibiting physicians from asking about firearm ownership is currently under review, ${ }^{39}$ and includes a number of rural practices, which is important given evidence that suicide rates among adolescents and adults are higher in rural versus urban communities and rural-urban disparities are increasing over time. ${ }^{3640}$

\section{Aim 1}

\section{Rationale}

We will survey clinical leadership and providers of paediatric primary care practices within the two systems of interest to understand variation in acceptability and use of the three firearm components that comprise the Safety Check intervention.

\section{Firearm safety intervention}

The Safety Check intervention is an evidence-based safety intervention targeted to parents of children aged $2-11 .{ }^{19}$ It includes three firearm components: screening, brief counselling and provision of firearm locks. Given evidence that providers perceive components of evidence-based practices differently, ${ }^{41}$ we have elected to examine the acceptability and use of each of the three individual components separately rather than ask questions about the intervention as a whole. Additionally, in aim 1, we are hoping to extend research on Safety Check by understanding acceptability and use in youth for suicide prevention. In the original study ${ }^{19}$ examining the efficacy of Safety Check, the intervention was conducted on a different age group, with a focus on safe firearm storage for injury prevention; the firearm intervention was bundled with other injury prevention strategies (eg, use of time out). This will allow us to understand to what extent Safety Check adaptations are needed to maximise the success of broad health systems implementation efforts.

\section{Sample}

The target population for this survey will be clinical leadership and providers at all paediatric primary care practices within the participating MHRN sites. Current estimates suggest that the number of practices across both systems is 82 . We elected to recruit all leaders (ie, physician managers) of paediatric primary care practices and all physicians who had seen 100 or more youth patients within the preceding 1 year from each of the 82 practices in the two health systems $(\mathrm{n}=261$; we anticipate $80 \%$ recruitment rate for a total of 209$).{ }^{42}$ A brief electronic survey will be sent to these clinical leaders and providers to determine acceptability and use of the three firearm components of the Safety Check intervention at that site. The required elements of informed consent will be described on the first page of the electronic survey and, if they agree to participate, clinical leadership and providers will consent by proceeding to the second page of the electronic survey. We have obtained a waiver of written documentation of consent from the University of Pennsylvania Institutional Review Board (IRB). To ascertain our sample, investigators at each site will identify the physician managers of paediatric practices within their system and will use electronic health records to identify primary care providers who treat youth in those practices. To enhance participation, and based on previous work suggesting that participation is very high if the request comes from a contact within the system, investigators at each site will initiate contact with potential participants at their site and send the web-based survey. Each participant who completes the survey will receive a $\$ 10$ gift card.

\section{Data sources}

\section{Primary data}

The survey will query about acceptability and use of the three firearm components of Safety Check, as well as relevant demographic information (eg, practice experience, firearm ownership). Sample questions include 'Asking all caregivers about the presence of firearms in the home would be an acceptable suicide prevention strategy in my practice' (rated from $1=$ strongly disagree to $6=$ strongly agree) and 'How often do you screen caregivers of youth for the presence of a firearm in the home of youth' (rated from $0=$ never to $4=$ always). All of the items in the survey have undergone cognitive response testing with physician managers and physician providers in paediatric primary care to identify ambiguous wording, awkward instructional sets and portions that may have been difficult to complete. ${ }^{42}$ Respondents have also evaluated the face validity of the survey during this process. In addition, before and after piloting, content validity was evaluated by experts in suicide research and primary care. 


\section{Secondary data}

We will use publicly available data sources including data from the MHRN data warehouse, which includes a combination of electronic health records, insurance claims data and the National Center for Health Statistics National Vital Statistics System. ${ }^{42}$ A programmer from each MHRN system will extract information from the data warehouse for participating providers: patient demographics (age, gender, race, ethnicity, patient language, Patient Health Questionnaire-9 (PHQ-9) ${ }^{43}$ score and diagnostic codes); encounters (e-codes related to suicide and self-inflicted injury) ${ }^{42}$; provider demographics (specialty, age, gender, race, ethnicity and year graduated); census (socioeconomic indicators for patients based on geocoded patient addresses); public census data (education, income and poverty); and mortality (patient death by suicide; this is only currently available in HFHS). Additionally, we will extract youth (aged 12-24) suicide deaths over the past 5 years at the county level for each primary care practice location from the National Vital Statistics System. ${ }^{42}$ These data will be used as predictor variables in analyses examining characteristics associated with acceptability and use of firearm safety strategies.

\section{Analyses}

Our quantitative analyses will identify the patient, provider and site characteristics associated with acceptability and use of the three components. To examine the effect of patient, provider and site factors on acceptability, we will use three separate ordinary least squares regression models. For each model, the dependent variable will be the mean of the acceptability items on the survey, measured on a 6-point scale. The independent variables will include measures of patient demographics (eg, ethnicity/race), enrolment, provider demographics (eg, age) and geographically derived socioeconomic and mortality indicators. Analyses will be conducted separately for the physician manager and physician provider samples when questions were worded differently (eg, 'I would consider using this strategy in my practice' for physicians and 'I would consider asking the providers in my practice to use this strategy' for managers). For questions that are worded identically (eg, 'Caregivers in my practice would view this strategy as intrusive'), we will run a single model and include responder type as a covariate.

In the physician manager group, respondents will report the per cent of providers in the practice who make use of each component. We will use linear regression models to examine the extent to which the independent variables predict use of the three components in paediatric practices. With 77 survey responses in each group, our models will have $80 \%$ power to detect a moderate effect $\left(\mathrm{r}^{2}=0.09\right)$ of the independent variable while controlling for seven covariates. In the provider sample we will use logistic regression to predict any use (vs no use) of the three practices. Assuming 77 survey responses and that 24\% of providers endorse use of each Safety Check component, we will be fully powered to detect between-group differences of $11 \%$ vs $36 \%$ in use of any of the three firearm components of Safety Check. For both samples, we will use multinomial logistic regression to model, among responders who report any use of each component at their site, the frequency with which they are used (rarely, sometimes, always). All models will control for health system.

\section{Outcome}

Based on the results generated from our survey, we will create a summary of acceptability and use of the three firearm components of Safety Check across each system to present as part of aim 2 to stakeholders.

\section{Aim 2}

In collaboration with MHRN stakeholders in these two systems, we will use intervention mapping and the CFIR ${ }^{24}$ to systematically develop and evaluate a multilevel menu of implementation strategies for a firearm safety intervention as a suicide prevention strategy in paediatric primary care.

\section{Rationale}

While intervention mapping has been proposed as a method for designing implementation strategies, ${ }^{42}$ it has yet to be widely used in this way. In a recently published proof-of-concept study, intervention mapping was employed to develop a multilevel implementation strategy to increase physicians' adherence to guidelines for depression. ${ }^{42}$ Compared with implementation as usual, the implementation strategy developed using intervention mapping was found to increase adherence to the guidelines. ${ }^{42}$ In the proposed study, we are applying these tools to the development of multilevel implementation strategies to change provider, organisation and system behaviours around firearm safety promotion practices in paediatric primary care. The approach is consistent with the CFIR. ${ }^{24}$ To accomplish this, we will focus on the fifth step of intervention mapping, which focuses on planning for adoption, implementation and sustainment. ${ }^{44}$

\section{Procedure}

The target behaviour is implementation of a firearm safety intervention in paediatric primary care intended to be a suicide prevention strategy. As in other studies, the final content to emerge from intervention mapping cannot yet be specified because it depends on information gleaned from the intervention mapping process.

\section{Needs assessment}

Using the CFIR to guide us, we will assess the needs of stakeholders who will be impacted by implementing a firearm safety intervention in paediatric primary care. ${ }^{24}$ We will conduct semistructured interviews with MHRN stakeholders from the following groups: (1) parents of youth ages 12-24, (2) physician providers, (3) non-physician providers (nurses, physician assistants, medical assistants), (4) leaders of primary care practices, (5) leaders of behavioural health, (6) leaders of quality 
improvement in each system, (7) system leaders, (8) third-party payers and (9) members of national credentialling bodies and professional organisations (eg, American Academy of Pediatrics). We will use purposive sampling ${ }^{45}$ to recruit approximately six individuals from each stakeholder group (three per health system) and sample across the two systems to ensure diversity in responses. We will also return to aim 1 participants and invite their participation and ask them who else in their system we should interview. We estimate we will conduct approximately 54 interviews; however, this number will depend on thematic saturation (ie, when no new ideas or themes are identified ${ }^{46}$ in subsequent interviews). We will use purposive sampling to recruit a sample of parents that includes at least $67 \%$ firearm owners. Fifty per cent of those firearm-owning parents will also be parents of youth at high risk for suicide based on medical records (ie, PHQ-9 scores). In the case of providers and leaders of primary care practices, we will use stratified sampling to ensure that we recruit participants endorsing use of at least one of the Safety Check firearm components as reported in aim 1 and at least $50 \%$ firearm owners as reported in aim 1. In part I of the interview, we will present practice stakeholders with the aggregated data on their system collected in aim 1 and use the qualitative interviews as an opportunity to understand these data in a more nuanced fashion (quan $\rightarrow$ QUAL), ${ }^{47}$ consistent with previous studies. ${ }^{48}$ For example, 'Your practice reported that about $50 \%$ of providers at your site screen for firearms in the home. Can you tell me more about how this is usually done?' In part II of the interview, we will use the $\mathrm{CFIR}^{24}$ to query around barriers and facilitators at the intervention, provider, inner and outer setting levels, and the support needed to be able to provide a firearm safety intervention in daily practice (eg, 'What kinds of changes or alterations to the intervention do you think would be needed for you to use it effectively?' and 'What resources or support would be needed to effectively implement screening, counseling, and gun lock provision in your setting?'). In part III of the interview, we will use intervention mapping to guide questions that identify programme adopters, implementers and sustainers, as well as the specific behaviours that each set of stakeholders needs to engage in to adopt, implement and sustain the Safety Check components (eg, 'Who, specifically, in your health system would need to make the decision to implement this intervention with all guardians of adolescents presenting for well and/or sick visits at your site'). Participants will be paid $\$ 25$ for participating in individual interviews.

We will recruit stakeholders using methods described in a similar MHRN study. ${ }^{42}$ To sample parents of youth, investigators at each site will identify random samples of 100 English-speaking parents or legal guardians of youth aged 12-24 who visited a primary paediatric care site within the previous 3 months from the electronic health records. Guardians will be sent an invitation letter with an opt-out card with postage. After 10 days, participants who have not returned the opt-out card will be called by phone, asked if they own a firearm (for sampling purposes) and invited to participate in our study. To sample providers, clinical leadership in paediatric primary care and behavioural health, leaders of quality improvement and system leaders, investigators at each site will identify the appropriate individuals to contact within their system, personally reach out to each of these individuals and invite them to participate via phone or email. We will work with clinical leadership in paediatric primary care to email invitations to providers inviting them to participate.

Interviews will be conducted by phone, digitally recorded with the participants' permission, professionally transcribed and loaded into NVivo software for data management and analysis. Interviewers include doctoral-level researchers who have received extensive training and supervision in qualitative interviewing, and bachelor's-level and master's-level research staff trained and closely supervised by the doctoral-level members of the research team. Analysis will be guided by integrated theory, which incorporates inductive and deductive features. ${ }^{49}$ This approach uses an inductive process of iterative coding to identify recurrent themes, categories and relationships in qualitative data. A comprehensive coding scheme is then developed on the basis of this analysis and applied to the data in order to produce a fine-grained descriptive analysis. A priori codes informed by CFIR $^{24}$ will also be applied (ie, deductive approach), such as coding for barriers and facilitators at the intervention, provider, and inner and outer setting levels. The research team will separately code a sample of the transcripts and compare their application of the coding scheme to assess the reliability and robustness of the coding scheme. Any disagreements in coding will be resolved through team discussion. We will also compute inter-rater agreement (ie, kappa), which provides a complementary approach to determining agreement between raters. ${ }^{46}$ We will ensure that reliability between raters is at least 0.80 or higher.

\section{Implementation strategies}

We will delineate implementation strategies based on existing theory, the scientific literature and the information gleaned from our stakeholder interviews. Consistent with the intervention mapping process, these implementation strategies will be translated into practical strategies using a theory-based causal model. We hypothesise that a model that accounts for individual behaviour and the environment would be most salient here, such as the theory of planned behaviour or social cognitive theory. ${ }^{5051}$ Using whatever theory(s) best fit the identified determinants for the intervention, individual, inner and outer settings, we will then delineate practical strategies which will become the multilevel menu of implementation strategies. For example, if providers are unfamiliar with firearm safety counselling, training may be identified as a promising implementation strategy, whereas if providers are uncomfortable with implementing firearm safety counselling (ie, low self-efficacy), specific intervention to improve 
their self-efficacy is warranted. If parents report that the intervention would seem off-putting if not done in the context of other safety conversations, an approach that bundles firearm safety conversations with promotion of other safe practices (eg, use of bicycle helmets) may be appropriate.

\section{Evaluation of feasibility and acceptability}

We will evaluate the multilevel menu of implementation strategies by returning to the initial stakeholders who completed the needs assessment and asking them to complete a brief survey assessing feasibility and acceptability. These participants will be paid an additional $\$ 25$ for completing this survey. We will also present our findings to leadership of each MHRN site and have them rate the feasibility and acceptability of the strategies. This will allow us to generate a list of the most feasible and acceptable implementation strategies within each level, which will then be tested in a larger effectiveness implementation trial. ${ }^{52}$

\section{DISCUSSION \\ Innovation}

There are conceptual and methodological innovations in the proposed study, the former referring to the potential to directly impact youth with suicidal thoughts and behaviours. Specifically, this study seeks to understand how to implement a universal firearm safety intervention in primary care. Previous studies have used targeted intervention approaches. ${ }^{53}$ However, youth experiencing suicidal thoughts and behaviours often do not access services during times of heightened risk, ${ }^{54}$ suggesting a universal approach has the potential to reach more youth, and ultimately reduce suicide deaths. Addressing a highly prevalent social burden and understanding how health systems and their patients perceive a minimally intrusive yet common sense approach to improving firearm safety have the potential to improve patient quality of life while better tailoring limited healthcare resources. Methodological innovation refers to the impact of the proposed work on systematic and rigorous methods to develop implementation strategies. To date, few studies have used intervention mapping to develop a multilevel menu of implementation strategies. ${ }^{4556}$ Further, this study will use the CFIR ${ }^{24}$ as part of this process to develop implementation strategies at the individual (eg, providers), inner setting (eg, paediatric practices), outer setting, and intervention characteristics levels. Evidence from the health services literature suggests that multilevel implementation strategies may be more effective than single-level implementation strategies. ${ }^{57-59}$

\section{Limitations}

Despite the strengths of the current study, several limitations should be noted. A primary limitation is that the study will be conducted in two health systems in the USA, and the extent to which results will generalise to other health systems is unknown. Moreover, both health systems are a part of the MHRN; health systems participating in the MHRN may have unique characteristics that may limit the generalisability of study findings to other healthcare systems (eg, an existing infrastructure to support partnerships with external researchers). Nonetheless, the diversity of the two systems, which are located in two distinct geographical and cultural settings, may mitigate threats to external validity. In addition, the Safety Check intervention was initially developed and tested as a broader safety initiative for parents of young children in which firearm storage was one of several safety targets. As such, the effectiveness of Safety Check as a stand-alone firearm safety intervention specifically for suicide prevention in paediatric primary care remains unknown. Additionally, the effectiveness of the intervention has been established based on parental self-report. It is possible that some parents may not be forthcoming when discussing matters related to firearm storage practices. Similarly, it is possible that providers and/or practice leaders may be reluctant to disclose information related to firearm safety given the sensitive nature of the topic. In an effort to mitigate these potential concerns, we will speak to both firearm-owning and non-owning stakeholders when possible to obtain a range of perspectives. We will also seek to understand providers' concerns about parental reluctance to discuss firearm ownership and storage during qualitative interviews, as understanding these concerns will be important in the development of implementation strategies. However, this is not our primary focus; other ongoing work $^{6061}$ explores the perspectives of firearm owners in greater depth.

\section{Impact}

The work proposed has the potential to impact public health in two important ways. First, it will be a critical step in the research agenda of reducing death by suicide in children and adolescents by engendering a better understanding of how to implement an evidence-based firearms safety intervention, specifically components of Safety Check, in paediatric primary care. Second, it will advance the implementation science literature by providing a process, informed by the CFIR, ${ }^{24}$ for how to systematically develop implementation strategies in partnership with stakeholders across multiple levels of implementation (ie, providers, inner and outer contexts).

\section{ETHICS AND DISSEMINATION}

Study procedures have been approved by the University of Pennsylvania (Protocol number 824449). The IRBs of HFHS and BSW have ceded review to the University of Pennsylvania IRB. We began data collection for this project in August 2016. Results of aims 1 and 2 will be submitted for publication in peer-reviewed journals.

Author affiliations

${ }^{1}$ Department of Psychiatry, University of Pennsylvania Perelman School of Medicine, Philadelphia, Pennsylvania, USA 
${ }^{2}$ School of Social Policy and Practice, University of Pennsylvania, Philadelphia, Pennsylvania, USA

${ }^{3}$ Henry Ford Health System, Center for Health Policy and Health Services Research and Behavioral Health Services, Detroit, Michigan, USA

${ }^{4}$ Center for Applied Health Research, Baylor Scott \& White Health, jointly with Central Texas Veterans Health Care System, Dallas, Texas, USA

${ }^{5}$ Department of Pediatrics, University of Pennsylvania Perelman School of Medicine, Philadelphia, Pennsylvania, USA

${ }^{6}$ Division of Emergency Medicine, The Children's Hospital of Philadelphia, Philadelphia, Pennsylvania, USA

Correction notice This paper has been amended since it was published Online First. Owing to a scripting error, some of the publisher names in the references were replaced with 'BMJ Publishing Group'. This only affected the full text version, not the PDF. We have since corrected these errors and the correct publishers have been inserted into the references.

Acknowledgements We are especially grateful for the support and cooperation the Mental Health Research Network has provided for this project, particularly Gregory Simon, MD, MPH, and the MHRN Suicide Prevention SIG. We would like to thank the following experts who provided their time and input on this project: Dr Collen Barry, Dr Shari Barkin, Dr Maria Fernandez, Dr Carol Runyan, Dr Garen Wintemute, Dr Marian Betz and Dr Megan Ranney. We are also grateful for the help provided by Danielle R Adams, BA; Kelly Zentgraf, BA; and Courtney Gregor, BA in assisting with this project.

Contributors RSB is the principal investigator for the study protocol. RSB generated the idea and designed the study, and approved all changes. CBW was the primary writer of the manuscript and is the project director for the study. CBW, SJH, SCM, BKA, JEZ, JAF, GKB, AL and RRB all made substantial contributions to study conception and design. All authors reviewed and provided feedback for this manuscript. The final version of this manuscript was vetted and approved by all authors.

Funding This project is supported by the following grants from NIMH: R21 MH109878-01 (RSB), F32 MH103955 (CBW) and U19 MH092201 (MHRN).

Competing interests None declared.

Patient consent Not applicable.

Ethics approval University of Pennsylvania IRB.

Provenance and peer review Not commissioned; externally peer reviewed.

Open Access This is an Open Access article distributed in accordance with the Creative Commons Attribution Non Commercial (CC BY-NC 4.0) license, which permits others to distribute, remix, adapt, build upon this work non-commercially, and license their derivative works on different terms, provided the original work is properly cited and the use is non-commercial. See: http://creativecommons.org/ licenses/by-nc/4.0/

(c) Article author(s) (or their employer(s) unless otherwise stated in the text of the article) 2017. All rights reserved. No commercial use is permitted unless otherwise expressly granted.

\section{REFERENCES}

1. Curtin SC, Warner M, Hedegaard H. Increase in Suicide in the United States, 1999-2014. NCHS Data Brief 2016:1-8.

2. National Center for Health Statistics. 10 leading causes of death by age group, United States (2014). Atlanta, GA: Centers for Disease Control and Prevention, National Center for Injury Prevention and Control, 2014

3. Miller M, Azrael D, Barber C. Suicide mortality in the United States: the importance of attending to method in understanding populationlevel disparities in the burden of suicide. Annu Rev Public Health 2012;33:393-408.

4. Hepburn L, Miller M, Azrael D, et al. The US gun stock: results from the 2004 national firearms survey. Inj Prev 2007;13:15-19.

5. Kalesan B, Villarreal MD, Keyes KM, et al. Gun ownership and social gun culture. Inj Prev 2016;22:216-20.

6. Barber CW, Miller MJ. Model for estimating reduction in U.S. suicide deaths following a reduction in suicidal adult persons' access to firearms. Rockville, MD:National Institute of Mental Health and Research Prioritization Task Force, 2014.

7. Kreitman N. The coal gas story. United Kingdom suicide rates, 196071. Br J Prev Soc Med 1976;30:86-93.
8. Gunnell D, Middleton N, Frankel S. Method availability and the prevention of suicide--a re-analysis of secular trends in England and Wales 1950-1975. Soc Psychiatry Psychiatr Epidemiol 2000;35:437-43.

9. Gunnell D, Fernando R, Hewagama M, et al. The impact of pesticide regulations on suicide in Sri Lanka. Int J Epidemiol 2007;36:1235-42.

10. Lubin G, Werbeloff N, Halperin D, et al. Decrease in suicide rates after a change of policy reducing access to firearms in adolescents: a naturalistic epidemiological study. Suicide Life Threat Behav 2010;40:421-4.

11. Mann JJ, Michel CA. Prevention of firearm suicide in the United States: what works and what Is possible. Am J Psychiatry 2016;173:969-79.

12. Webster DW, Vernick JS, Zeoli AM, et al. Association between youth-focused firearm laws and youth suicides. JAMA 2004;292:594-601.

13. Barber CW, Miller MJ. Reducing a suicidal person's access to lethal means of suicide: a research agenda. Am J Prev Med 2014;47(3 Suppl 2):S264-72.

14. Hogan MF, Grumet JG. Suicide prevention: an emerging priority for health care. Health Aff 2016;35:1084-90.

15. Glenn CR, Franklin JC, Nock MK. Evidence-based psychosocial treatments for self-injurious thoughts and behaviors in youth. $J$ Clin Child Adolesc Psychol 2015;44:1-29.

16. Luoma JB, Martin CE, Pearson JL. Contact with mental health and primary care providers before suicide: a review of the evidence. Am J Psychiatry 2002;159:909-16.

17. Husky MM, Olfson M, He JP, et al. Twelve-month suicidal symptoms and use of services among adolescents: results from the National Comorbidity Survey. Psychiatr Serv 2012;63:989-96.

18. Klein JD, Wilson KM, McNulty M, et al. Access to medical care for adolescents: results from the 1997 Commonwealth Fund Survey of the Health of Adolescent Girls. J Adolesc Health 1999;25:120-30.

19. Barkin SL, Finch SA, Ip EH, et al. Is office-based counseling about media use, timeouts, and firearm storage effective? Results from a cluster-randomized, controlled trial. Pediatrics 2008;122:e15-e25.

20. Dowd MD, Sege RD. Council on Injury, Violence, and Poison Prevention Executive Committee American Academy of Pediatrics. Firearm-related injuries affecting the pediatric population. Pediatrics 2012;130:e1416-23.

21. Leshner Al, Altevogt BM, Lee AF, et al. Priorities for research to reduce the threat of firearm-related violence. Washington, DC: Institute of Medicine and National Research Council, 2013.

22. Roszko PJ, Ameli J, Carter PM, et al. Clinician attitudes, screening poractices, and interventions to reduce firearm-related injury. Epidemiol Rev 2016;38:mxv005-110.

23. Betz ME, Wintemute GJ. Physician counseling on firearm safety: a new kind of cultural competence. JAMA 2015;314:449-50.

24. Damschroder LJ, Aron DC, Keith RE, et al. Fostering implementation of health services research findings into practice: a consolidated framework for advancing implementation science. Implement Sci 2009;4:50.

25. Tabak RG, Khoong EC, Chambers DA, et al. Bridging research and practice: models for dissemination and implementation research. Am J Prev Med 2012;43:337-50.

26. Finch SA, Weiley V, Ip EH, et al. Impact of pediatricians' perceived self-efficacy and confidence on violence prevention counseling: a national study. Matern Child Health J 2008;12:75-82.

27. Ozer EM, Adams SH, Gardner LR, et al. Provider self-efficacy and the screening of adolescents for risky health behaviors. J Adolesc Health 2004;35:101-7.

28. Maiuro RD, Vitaliano PP, Sugg NK, et al. Development of a health care provider survey for domestic violence: psychometric properties. Am J Prev Med 2000;19:245-52.

29. Cheng TL, DeWitt TG, Savageau JA, et al. Determinants of counseling in primary care pediatric practice: physician attitudes about time, money, and health issues. Arch Pediatr Adolesc Med 1999;153:629-35.

30. Wintemute GJ, Betz ME, Ranney ML. Yes, You Can: Physicians, Patients, and Firearms. Ann Intern Med 2016;165:205-13.

31. BetzME, Ranney ML, Wintemute GJ. Physicians, patients and firearms: the courts say "yes" [published online March 7 2017]. Ann Intern Med 2017

32. Schaafsma D, Stoffelen JM, Kok G, et al. Exploring the development of existing sex education programmes for people with intellectual disabilities: an intervention mapping approach. J Appl Res Intellect Disabil 2013;26:157-66

33. Belansky ES, Cutforth N, Chavez R, et al. Adapted intervention mapping: a strategic planning process for increasing physical activity and healthy eating opportunities in schools via environment and policy change. J Sch Health 2013;83:194-205. 
34. Fernández ME, Gonzales A, Tortolero-Luna G, et al. Using intervention mapping to develop a breast and cervical cancer screening program for Hispanic farmworkers: Cultivando La Salud. Health Promot Pract 2005;6:394-404.

35. Hurley DA, Murphy LC, Hayes D, et al. Using intervention mapping to develop a theory-driven, group-based complex intervention to support self-management of osteoarthritis and low back pain (SOLAS). Implement Sci 2016;11:56.

36. Bartholomew LK, Parcel G, Gottlieb NH. Intervention mapping: designing theory and evidence-based health promotion programs. Mountain View, CA: Mayfield Publishing, 2001.

37. Waltz TJ, Powell BJ, Chinman MJ, et al. Expert Recommendations for Implementing Change (ERIC): protocol for a mixed methods study. Implement Sci 2014;9:39.

38. Bridge JA, Asti L, Horowitz LM, et al. Suicide Trends Among Elementary School-Aged Children in the United States From 1993 to 2012. JAMA Pediatr 2015;169:673.

39. Texas Legisislature. An act relating to prohibiting certain physician questions regarding firearms. HB 2823. 2823, 2015.

40. Fontanella CA, Hiance-Steelesmith DL, Phillips GS, et al. Widening rural-urban disparities in youth suicides, United States, 1996-2010. JAMA Pediatr 2015;169:466-73.

41. Pellecchia M, Connell JE, Beidas RS, et al. Dismantling the active ingredients of an intervention for children with autism. J Autism Dev Disord 2015;45:2917-27.

42. Rahm AK, Boggs JM, Martin C, et al. Facilitators and barriers to implementing Screening, Brief Intervention, and Referral to Treatment (SBIRT) in primary care in integrated health care settings. Subst Abus 2015;36:281-8.

43. Kroenke K, Spitzer RL, Williams JB. The PHQ-9: validity of a brief depression severity measure. J Gen Intern Med 2001;16:606-13.

44. Bartholomew L, Markham CM, Ruiter RAC, et al. Planning health promotion programs: an intervention mapping approach. San Francisco, CA: Jossey-Bass, 2016.

45. Palinkas LA, Horwitz SM, Green CA, et al. Purposeful sampling for qualitative data collection and analysis in mixed method implementation research. Adm Policy Ment Health 2015;42:533-44.

46. Hays DG, Singh AA. Qualitative inquiry in clinical and educational settings. New York, NY: The Guilford Press, 2012.

47. Palinkas LA, Aarons GA, Horwitz S, et al. Mixed method designs in implementation research. Adm Policy Ment Health 2011;38:44-53.

48. Aarons GA, Palinkas LA. Implementation of evidence-based practice in child welfare: service provider perspectives. Adm Policy Ment Health 2007;34:411-9.
49. Bradley EH, Curry LA, Devers KJ. Qualitative data analysis for health services research: developing taxonomy, themes, and theory. Health Serv Res 2007;42:1758-72.

50. Bandura A. Human agency in social cognitive theory. Am Psychol 1989:44:1175-84.

51. Fishbein M. Developing effective behavior change interventions: some lessons learned from behavioral research. NIDA Res Monogr 1995;155:225-46.

52. Curran GM, Bauer M, Mittman B, et al. Effectiveness-implementation hybrid designs: combining elements of clinical effectiveness and implementation research to enhance public health impact. Med Care 2012;50:217-26.

53. Johnson RM, Frank EM, Ciocca M, et al. Training mental healthcare providers to reduce at-risk patients' access to lethal means of suicide: evaluation of the CALM Project. Arch Suicide Res 2011;15:259-64.

54. Yip PS, Caine E, Yousuf $\mathrm{S}$, et al. Means restriction for suicide prevention. Lancet 2012;379:2393-9.

55. Donaldson A, Poulos RG. Planning the diffusion of a neck-injury prevention programme among community rugby union coaches. $\mathrm{Br} J$ Sports Med 2014:48:151-9.

56. Donaldson A, Lloyd DG, Gabbe BJ, et al. We have the programme, what next? Planning the implementation of an injury prevention programme. Injury Prevention 2016:injuryprev-2015-041737-8.

57. Evans D, Mellins R, Lobach $\mathrm{K}$, et al. Improving care for minority children with asthma: professional education in public health clinics. Pediatrics 1997;99:157-64.

58. Glanz K, Bishop DB. The role of behavioral science theory in development and implementation of public health interventions. Annu Rev Public Health 2010;31:399-418.

59. Kok G, Schaalma H, Ruiter RA, et al. Intervention mapping: protocol for applying health psychology theory to prevention programmes. $J$ Health Psychol 2004;9:85-98.

60. Barber C, Frank E, Demicco R. Reducing suicides through partnerships between health professionals and gun owner groupsbeyond docs vs glocks. JAMA Intern Med 2017;177:5-6.

61. Betz ME, Azrael D, Barber $\mathrm{C}$, et al. Public opinion regarding whether speaking with patients about firearms is appropriate. Ann Intern Med 2016;165:543.

62. Damschroder L. Advanced topics for implementation science research: pragmatic application and scientific advancement of the consolidated frame work for implementation research (CFIR) Advanced Topics in Implementation Science (IS) Research Webinar Series. 